\title{
Watermark Detection for Noisy Interpolated Images
}

\author{
Alexia Giannoula, Nikolaos V. Boulgouris, Member, IEEE, Dimitrios Hatzinakos, Senior Member, IEEE, and \\ Konstantinos N. Plataniotis, Senior Member, IEEE
}

\begin{abstract}
In this brief, the case where the watermark is detected in a noisy interpolated version of the originally watermarked image is investigated. Polyphase decomposition is utilized at the detection side in order to enable the flexible formation of a fused image, which is appropriate for watermark detection. The optimal fused correlator, obtained by combining information from different image components, is derived through a statistical analysis of the correlation detector properties, followed by Lagrange optimization. It is shown that it is preferable to perform detection in a fused image rather than the original image.
\end{abstract}

Index Terms-Noisy images, watermark detection.

\section{INTRODUCTION}

I N MANY IMAGE processing scenarios, it would be desirable to embed a watermark on an image right after acquisition in order to ensure that no unwatermarked version of the original image is stored or distributed. In such cases, the image might later undergo image processing operations before storage or distribution. In other cases, an attacker might perform image scaling as part of his malicious attack. In the very common case of image resizing at dimensions larger than the original, the watermark information, which was embedded in the low-resolution version upon acquisition, is inevitably spread on the larger image. The larger image could then be compressed and transmitted to any potential recipients (in a lawful distribution scenario) or distorted in the case of a malicious attack.

Most of the recent watermark detection methods either try to restore the original image in order to regain resynchronization with the initially embedded watermark or attempt to estimate an appropriate "synchronized" watermark, by subjecting the watermark signal to a sequence of identical operations (such as those applied on the watermarked image) [1]-[3]. In all cases, robust watermarking techniques should be employed in order to enable estimation of the distortions that the image has undergone after watermark embedding. Surprisingly, improving on the aforementioned techniques in the noisy-case scenario, by exploiting the information generated by interpolating the watermarked image, has not been investigated.

In this brief, an efficient method is introduced and it is demonstrated that the watermark can be optimally detected in the interpolated domain rather than the original image domain. Two fundamental assumptions have been adopted:

Manuscript received April 27, 2005. This work was supported by the Natural Sciences \& Engineering Research Council of Canada (NSERC). This paper was recommended by Associate Editor Y. Q. Shi.

A. Giannoula, D. Hatzinakos, and K. N. Plataniotis are with the Electrical and Computer Engineering Department, University of Toronto, Toronto, ON M5S 3G4, Canada (e-mail: alexia@ comm.toronto.edu).

N. V. Boulgouris is with the Department of Electronic Engineering, Division of Engineering, King's College London, London WC2R 2LS, U.K.

Digital Object Identifier 10.1109/TCSII.2006.870213
1) the originally watermarked image has been upscaled; 2) the upscaled image has been subsequently distorted by noise, compression, etc, (a robust watermarking technique is assumed to be applied for estimating the scaling factor). The efficiency of the proposed scheme is based on a procedure that takes place prior to detection. Specifically, at the detection side, linear finite-impulse response (FIR) filters are applied in order to derive several estimates of the original (low-resolution) watermarked image, based on the noisy interpolation samples. In this way, a fused image is generated by combining different filtered components of the interpolated image. A statistical analysis of the properties of the proposed fused correlation detector, applied on the aforementioned fused image, is undertaken in this brief for the case of additive random watermarks and the optimal fusion is contemplated using Lagrange multipliers. It should be noted that the proposed scheme is not a full-fledged image watermarking technique, but it can be seen as an elegant methodology that can be applied in conjunction with robust watermarking techniques, in order to improve the accuracy of detection.

\section{PROBLEM FORMULATION}

Let $x[\boldsymbol{m}]$ be a $N_{1} \times N_{2}$ grayscale image, which will be considered the host signal. It has been assumed that $m$ corresponds to the pixel indexes and that $\boldsymbol{m} \in \mathcal{N}$, where the symbol $\mathcal{N}$ denotes the set of all $2 \times 1$ integer vectors (boldfaced letters represent matrices and vectors). The watermark sequence $w[\boldsymbol{m}]$ of size $N_{1} \times N_{2}$ is an i.i.d. Gaussian-distributed random pattern with zero mean value and variance $\sigma_{w}^{2}$, generated by a pseudorandom number generator using a suitable key. An additive embedding rule is employed, producing the watermarked image $x_{w}[\boldsymbol{m}]$

$$
x_{w}[\boldsymbol{m}]=x[\boldsymbol{m}]+p \cdot w[\boldsymbol{m}]
$$

where $p$ is a constant that controls the watermark embedding power.

An expanded image $x_{e}[\boldsymbol{m}]=x_{w}\left[\boldsymbol{M}^{-1} \boldsymbol{m}\right]$ is obtained, using a $2 \times 2$ nonsingular integer matrix $\boldsymbol{M}$ on the indexes $\boldsymbol{m}$ that define the set of all the vectors on the lattice generated by the sampling matrix $\boldsymbol{M}$ [4]. A linear low-pass filter $h_{I}(\boldsymbol{m})$ is assumed to be applied on $x_{e}[\boldsymbol{m}]$, generating an interpolated image $\widetilde{x}[\boldsymbol{m}]$. In this manner, the watermark signal, embedded in the low-resolution image, is linearly spread in the new pixel positions of the finer-resolution image $\widetilde{x}[\boldsymbol{m}]$. The eventual high-resolution image $s[\boldsymbol{m}]$, that will be used for detection, is assumed to be a noisy version of $\widetilde{x}[\boldsymbol{m}]$. Particularly, it is assumed that the distortion to which $\widetilde{x}[\boldsymbol{m}]$ is subjected can be modeled as additive noise, i.e.,

$$
s[\boldsymbol{m}]=\widetilde{x}[\boldsymbol{m}]+\widetilde{n}[\boldsymbol{m}]
$$




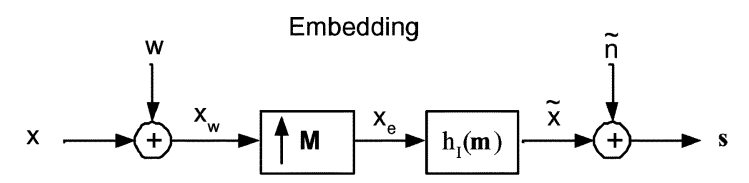

(a)

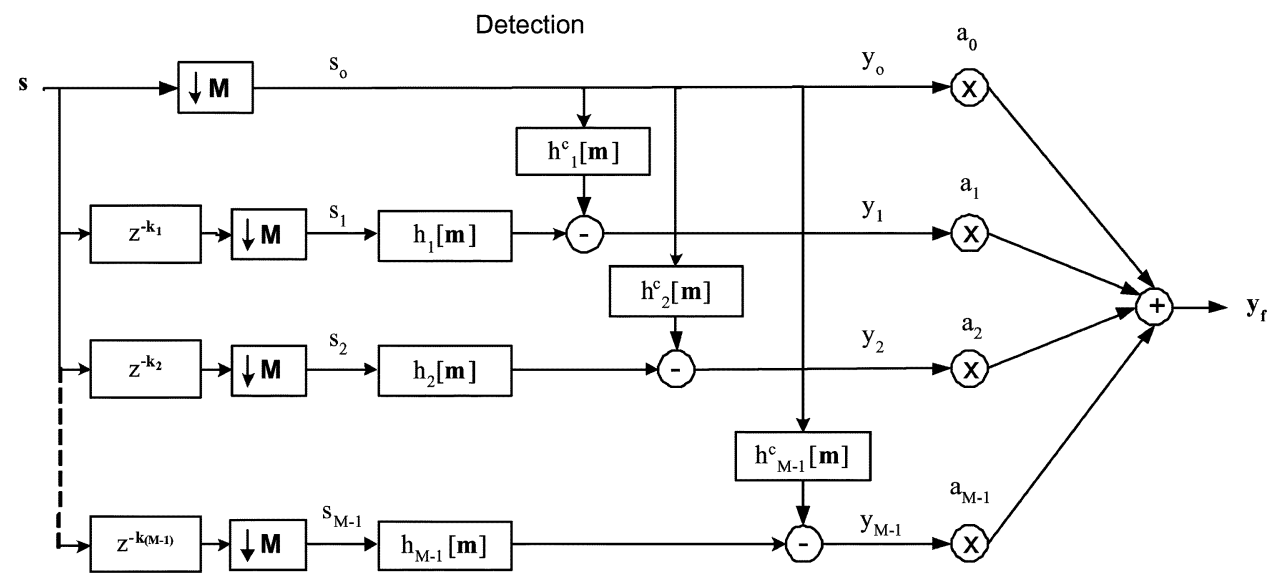

(b)

Fig. 1. Block diagram for (a) watermark embedding and (b) fused correlation detection.

where $\widetilde{n}[\boldsymbol{m}]$ is the noise signal. The embedding process is schematically described in Fig. 1(a).

The detection procedure will take place using the distorted high-resolution image $s[\boldsymbol{m}]$ in a way that exploits the additional watermark information due to interpolation. For this purpose, $s[\boldsymbol{m}]$ is initially decomposed into its constituent polyphase components [4]:

$$
s_{i}[\boldsymbol{m}]=s\left[\boldsymbol{M} \boldsymbol{m}+\boldsymbol{k}_{i}\right], \quad \boldsymbol{k}_{i} \in \mathcal{L}(\boldsymbol{M}) ; \quad i=0, \ldots, M-1
$$

where $\mathcal{L}(\boldsymbol{M})$ denotes the set of all integer vectors of the form $\boldsymbol{M} \boldsymbol{x}$ for $\boldsymbol{b} \in[0,1)^{2}$. For a given matrix $\boldsymbol{M}$, the number of vectors $\boldsymbol{k}_{i}$ is fixed and equal to $M=|\operatorname{det} \boldsymbol{M}|$. The polyphase components of the noisy interpolated image $s[\boldsymbol{m}]$ represent different sets of pixels. The zeroth polyphase component $s_{0}[\boldsymbol{m}]$ of $s[\boldsymbol{m}]$, obtained as shown in Fig. 1(b), essentially represents the original low-resolution watermarked image $x_{w}[\boldsymbol{m}]$ after noise corruption by a noise signal $n_{0}[\boldsymbol{m}]=\widetilde{n}_{0}[\boldsymbol{m}]$ (with variance $\sigma_{n_{0}}^{2}$ ), where $\widetilde{n}_{0}[\boldsymbol{m}]$ is the zeroth polyphase component of $\widetilde{n}[\boldsymbol{m}]$. The rest of the polyphase components $s_{i}[\boldsymbol{m}], i=1, \ldots, M-1$, represent interpolated pixels also corrupted by noise.

Our intention is to form $M$ low-resolution images $y_{i}[\boldsymbol{m}] i=$ $0, \ldots, M-1$, which will be different versions of $x_{w}[\boldsymbol{m}]$ and whose optimal fusion will provide a new image $y_{f}[\boldsymbol{m}]$, on which watermark detection will be more reliable than conventional detection on $s_{0}[\boldsymbol{m}]$. Since $s_{0}[\boldsymbol{m}]$ by itself is clearly a noisy version of $x_{w}[\boldsymbol{m}]$, it is used unaltered as $y_{0}[\boldsymbol{m}]$, i.e.,

$$
y_{0}[\boldsymbol{m}]=s_{0}[\boldsymbol{m}]=x_{w}[\boldsymbol{m}]+n_{0}[\boldsymbol{m}] .
$$

In addition, a sequence of estimates of $x_{w}[\boldsymbol{m}]$ are derived as follows: the generated polyphase output images $s_{i}[\boldsymbol{m}]$ (of dimensions $\left.N_{1} \times N_{2}\right), i=1, \ldots, M-1$, are first filtered using appropriate linear filters and then added up to produce

$$
y_{i}[\boldsymbol{m}]=x_{w}[\boldsymbol{m}]+n_{i}[\boldsymbol{m}]
$$

where $n_{i}[\boldsymbol{m}]$ is a linear combination of the $M$ noise signals which correspond to the components of $s[\boldsymbol{m}]$. The variance of the noise of each of the above images $y_{i}[m]$ is $\sigma_{n_{i}}^{2}$ and is usually greater than $\sigma_{n_{0}}^{2}$ due to the contribution of several noise terms during the derivation of $y_{i}[\boldsymbol{m}]$.

The proposed technique, subsequently, involves a fusion of the images $y_{0}[\boldsymbol{m}], y_{1}[\boldsymbol{m}], \ldots, y_{M-1}[\boldsymbol{m}]$ using the following rule:

$$
y_{f}[\boldsymbol{m}]=y_{f}[\boldsymbol{m}]=x_{w}[\boldsymbol{m}]+\sum_{i=0}^{M-1} a_{i} n_{i}[\boldsymbol{m}]
$$

where the weight coefficients $a_{i}, i=0, \ldots, M-1$ are real numbers confined in the $[0,1]$ interval and sum up to unity, i.e., $\sum_{i=0}^{M-1} a_{i}=1$. The fused image $y_{f}[\boldsymbol{m}]$ will be finally employed in the correlation detection described in Section III. The block diagram of both the embedding and detection procedures can be seen in Fig. 1.

For calculating each $y_{i}[\boldsymbol{m}](i \neq 0)$ two filters are utilized: filter $h[\boldsymbol{m}]$ to estimate $x_{w}[\boldsymbol{m}]$ and filter $h^{c}[\boldsymbol{m}]$ to cancel any undesirable interference, i.e., $y_{i}[\mathbf{m}]=h_{i}[\mathbf{m}] * s_{i}[\mathbf{m}]-h_{i}^{c}[\mathbf{m}] *$ $s_{0}[\mathbf{m}]$. It can be easily shown that practical choices for the $i$-th polyphase component of the respective filters $h[\mathbf{m}]$ and $h^{c}[\mathbf{m}]$ are

$$
h_{i}[\mathbf{m}]=b \cdot h_{I, i}[\mathbf{m}], \quad h_{i}^{c}[\mathbf{m}]=b h_{I, i}[\mathbf{m}] * h_{I, i}[\mathbf{m}]-\delta[\mathbf{m}]
$$

where $\delta[\boldsymbol{m}]$ is the Kronecker delta and $b$ is a scaling factor. ${ }^{1}$

\section{TheORETICAL PERFormance ANALYSIS}

The correlation detector will be undertaken in this brief, in order to examine whether a tested image, denoted by $y_{f, t}[\boldsymbol{m}]$, possibly contains a watermark or not. Correlation detectors have been widely used throughout the watermarking literature, such as in [5], [6]. A statistical binary hypothesis test is performed as follows.

\footnotetext{
${ }^{1}$ The factor $b$ can be adjusted so that $h_{i}^{c}[\mathbf{0}]=0$, for $i=1,2, \ldots, M-1$.
} 
- Hypothesis $H_{0}$ : the test image contains the watermark $w[\boldsymbol{m}]$.

- Hypothesis $H_{1}$ : the test image does not contain the watermark $w[\boldsymbol{m}]$.

Event $H_{1}$ occurs either if the test image is not watermarked (event $H_{1 a}$ ) or if it is watermarked with a different watermark $w_{d}[\boldsymbol{m}] \neq w[\boldsymbol{m}]$ (event $H_{1 b}$ ). The three events mentioned above, can be combined in the following expression for the test image:

$$
y_{f, t}[\boldsymbol{m}]=x[\boldsymbol{m}]+p \cdot w_{e}[\boldsymbol{m}]+\sum_{i=0}^{M-1} a_{i} n_{i}[\boldsymbol{m}]
$$

where the watermark $w[\boldsymbol{m}]$ is indeed embedded in the signal if $p \neq 0$ and $w_{e}[\boldsymbol{m}]=w[\boldsymbol{m}]$ (event $H_{0}$ ), and it is not embedded in the signal if $p=0$ (no watermark is present, event $H_{1 a}$ ) or $p \neq 0$ and $w_{e}[\boldsymbol{m}]=w_{d}[\boldsymbol{m}](\neq w[\boldsymbol{m}]$ ) (wrong watermark presence, event $H_{1 b}$ ).

The correlation between the image under investigation $y_{f, t}[\boldsymbol{m}]$ and the watermark sequence $w[\boldsymbol{m}]$ is quantitatively expressed by the correlator output

$c_{f}=\frac{1}{N_{1} N_{2}} \sum_{\mathbf{m}}\left(x[\boldsymbol{m}] w[\boldsymbol{m}]+p w[\boldsymbol{m}] w_{e}[\boldsymbol{m}]+w[\boldsymbol{m}] \sum_{i=0}^{M-1} a_{i} n_{i}[\boldsymbol{m}]\right)$.

In order to decide on the valid hypothesis, $c_{f}$ is compared against a suitably selected threshold $T$. The performance of such a correlation-based technique can be measured in terms of the probability of false alarm $P_{\mathrm{fa}}(T)$ and the probability of false rejection $P_{\mathrm{fr}}(T)$. For the interested reader, alternative detection schemes can be found in [7], [8].

For the pseudorandom watermarks employed in this brief, the Central Limit Theorem [9] can be applied, in order to establish that the involved correlator output pdfs under the two hypotheses, $f_{c_{f} \mid H_{0}}, f_{c_{f} \mid H_{1}}$, attain a Gaussian distribution. Therefore, these pdfs can be described by their mean $\mu_{c_{f} \mid H_{0}}, \mu_{c_{f} \mid H_{1}}$, and variance values $\sigma_{c_{f} \mid H_{0}}^{2}, \sigma_{c_{f} \mid H_{1}}^{2}$. Using (9), the mean value $\mu_{c_{f}}=E\left\{c_{f}\right\}$ of the correlation detector $(E\{\cdot\}$ denotes statistical expectation), under the above assumptions, can be evaluated [6]

$$
\mu_{c_{f}}=\frac{p}{N_{1} N_{2}} \sum_{\mathbf{m}} E\left\{w[\boldsymbol{m}] w_{e}[\boldsymbol{m}]\right\}
$$

Similarly, its variance $\sigma_{c_{f}}^{2}=E\left\{c_{f}^{2}\right\}-E^{2}\left\{c_{f}\right\}$ is

$$
\begin{aligned}
\sigma_{c_{f}}^{2}=\frac{1}{N_{1}^{2} N_{2}^{2}} & \\
& \times\left(\sum _ { \mathbf { m } } \left(E\left\{x^{2}[\boldsymbol{m}]\right\} E\left\{w^{2}[\boldsymbol{m}]\right\}\right.\right. \\
& +p^{2} E\left\{w^{2}[\boldsymbol{m}] w_{e}^{2}[\boldsymbol{m}]\right\} \\
& +2 p E\{x[\boldsymbol{m}]\} E\left\{w^{2}[\boldsymbol{m}] w_{e}[\boldsymbol{m}]\right\} \\
& \left.+E\left\{w^{2}[\boldsymbol{m}]\right\} \sum_{i} a_{i}^{2} E\left\{n_{i}^{2}[\boldsymbol{m}]\right\}\right) \\
& \left.+\sum_{\mathbf{m}} \sum_{\mathbf{r}, \mathbf{r} \neq \mathbf{m}} p^{2} E\left\{w[\boldsymbol{m}] w[\boldsymbol{r}] w_{e}[\boldsymbol{m}] w_{e}[\boldsymbol{r}]\right\}\right) \mu_{c_{f}}^{2} .
\end{aligned}
$$

Zero-mean watermarks have been treated and the statistical independence between the host signal $x[\boldsymbol{m}]$, the watermarks $w[\boldsymbol{m}], w_{e}[\boldsymbol{m}]$ and the noise signals $n_{i}[\boldsymbol{m}], i=0, \ldots, M-1$, has been taken into account in deriving the above formulas, as well as the fact that the watermark and the noise signals are i.i.d Gaussian-distributed with zero-mean. Equations(10) and (11) are general and can be applied to all three events, $H_{0}, H_{1 a}$, and $H_{1 b}$. Bearing in mind that $E\left\{w^{2}[\boldsymbol{m}]\right\}=\sigma_{w}^{2}, E\left\{w^{3}[\boldsymbol{m}]\right\}=0$ and $E\left\{w^{4}[\boldsymbol{m}]\right\}=3 \sigma_{w}^{4}$, for a zero-mean Gaussian watermark $w[\boldsymbol{m}]$ and assuming, also, wide-sense stationarity for the host image $x[\boldsymbol{m}]$, where $\mu_{x}=E\{x[\boldsymbol{m}]\}$ and $\sigma_{x}^{2}=E\left\{x^{2}[\boldsymbol{m}]\right\}-\mu_{x}^{2}$, analytical expressions for $\mu_{c_{f}}$ and $\sigma_{c_{f}}^{2}$ can be derived for all three events $H_{0}, H_{1 a}$, and $H_{1 b}$

$$
\begin{aligned}
& \mu_{c_{f}}= \begin{cases}p \cdot \sigma_{w}^{2}, & \text { event } H_{0} \\
0, & \text { event } H_{1 a} \\
0, & \text { event } H_{1 b}\end{cases} \\
& \sigma_{c_{f}}^{2}= \begin{cases}\frac{\sigma_{w}^{2}}{N_{1} N_{2}}\left(\mu_{x}^{2}+\sigma_{x}^{2}+2 p^{2} \sigma_{w}^{2}+\sum_{i=0}^{M-1} a_{i}^{2} \sigma_{n_{i}}^{2}\right), & \text { event } H_{0} \\
\frac{\sigma_{w}^{2}}{N_{1} N_{2}}\left(\mu_{x}^{2}+\sigma_{x}^{2}+\sum_{i=0}^{M-1} a_{i}^{2} \sigma_{n_{i}}^{2}\right), & \text { event } H_{1 a} \\
\frac{\sigma_{w}^{2}}{N_{1} N_{2}}\left(\mu_{x}^{2}+\sigma_{x}^{2}+p^{2} \sigma_{w}^{2}+\sum_{i=0}^{M-1} a_{i}^{2} \sigma_{n_{i}}^{2}\right), & \text { event } H_{1 b} .\end{cases}
\end{aligned}
$$

Comparing $\mu_{c_{f}}, \sigma_{c_{f}}^{2}$ with the corresponding values $\mu_{c}, \sigma_{c}^{2}$ in the distortion-free case, it can be readily found [6]

$$
\begin{aligned}
\mu_{c_{f}} & =\mu_{c} \\
\sigma_{c_{f}}^{2} & =\sigma_{c}^{2}+\frac{\sigma_{w}^{2}}{N_{1} N_{2}} \cdot \sum_{i=0}^{M-1} a_{i}^{2} \sigma_{n_{i}}^{2} .
\end{aligned}
$$

Since the mean value of the fused correlator is identical to that corresponding to the noiseless scenario (see (14)), the fused noise term of (15) can only affect the detection performance of the system. Therefore, this summation term is required to be minimized in order to reduce $\sigma_{c_{f}}^{2}$ and achieve a gain in the system detection reliability. For this reason, the optimal values $\left(a_{0}^{*}, a_{1}^{*}, \ldots, a_{M-1}^{*}\right)$, in the sense of minimizing $\sum_{i=0}^{M-1} a_{i}^{2} \sigma_{n_{i}}^{2}$, need to be determined. By following the Lagrangian approach [10], the global minimum can be derived as follows:

$$
\left(a_{0}^{*}, \ldots, a_{M-1}^{*}\right)=\left(\frac{1}{\sigma_{n_{0}}^{2} \sum_{k} \frac{1}{\sigma_{n_{k}}^{2}}}, \ldots, \frac{1}{\sigma_{n_{M-1}}^{2} \sum_{k} \frac{1}{\sigma_{n_{k}}^{2}}}\right) .
$$

The variance $\sigma_{n_{i}}^{2}$ of the noise of each estimate $y_{i}[\boldsymbol{m}]$ can be estimated by filtering the initial samples, i.e., $\tilde{s}_{i}[\mathbf{m}]=h_{I, i}[\mathbf{m}] *$ $s_{0}[\mathbf{m}]$ and subtracting the result from $s_{i}[\mathbf{m}]$ (details of the above optimization analysis have been omitted due to lack of space).

\section{EXPERIMENTAL RESULTS}

A large number of experiments were performed to illustrate the improvement in the detection performance of the proposed watermarking scheme. For this purpose, the $256 \times 256$ grayscale Lenna image was watermarked using additive embedding in the pixel domain. A watermark embedding power $p$ that resulted in watermarked images with peak-signal-to-noise-ratio 
(PSNR) approximately equal to $34.2 \mathrm{db}$, was used in all experiments. In order to keep the analysis simple, no masking properties of the human visual system (HVS) were considered and a constant embedding strength factor was assumed. The image was, subsequently, expanded using the following matrix:

$$
\boldsymbol{M}=\left[\begin{array}{ll}
2 & 0 \\
0 & 2
\end{array}\right] \text {. }
$$

In the sequel, the interpolation filter

$$
\begin{aligned}
H_{I}(\boldsymbol{z})=1+ & \frac{1}{2}\left(z_{0}^{-1}+z_{0}\right)+\frac{1}{2}\left(z_{1}^{-1}+z_{1}\right) \\
& +\frac{1}{4}\left(z_{0}^{-1} z_{1}+z_{0} z_{1}^{-1}+z_{0} z_{1}+z_{0}^{-1} z_{1}^{-1}\right)
\end{aligned}
$$

was employed ${ }^{2}$ in order to generate new image samples. The output is a $512 \times 512$ image, which is, finally, distorted by additive noise. The synthesis and cancellation post-filters, $h[\boldsymbol{m}]$ and $h^{c}[\boldsymbol{m}]$ respectively, were chosen as in (7), in order to produce $M$ (in our case $M=4$ ) estimates of the originally available (low-resolution) watermarked image. These estimates were fused using the optimal weights. The fused image, of dimensions $256 \times 256$, was finally examined for the presence of a potential watermark using a correlation detector.

All results presented in the remainder of this section, were obtained using $10^{6}$ Monte Carlo runs and the computation of the $R O C$ curves (plots of $P_{\mathrm{fa}}$ versus $P_{\mathrm{fr}}$ ) was performed under events $H_{0}$ and $H_{1 b}$, since the latter represents the worst case assumption (the image being watermarked with a different watermark). The ROC curves were generated both experimentally and theoretically. The theoretic derivation was based on the Gaussian model assumption for the correlation detector, whereas the experimental ROC curves were computed by evaluating the actual watermark detection performance over all Monte Carlo runs. The performance of the proposed scheme was compared against two traditional detection scenarios: (a) watermark detection on the noisy low-resolution image and (b) watermark detection on the noisy high-resolution (interpolated) image, using a watermark which was generated from the original watermark, by an interpolation process, identical to the process undergone by the image.

In a first set of experiments, additive i.i.d Gaussian noise with zero mean was added to the interpolated image and the watermark detection was performed using our optimally fused image or the two alternative aforementioned scenarios. Denoising was not applied prior to the evaluation of the investigated techniques. The theoretical and simulated ROC curves corresponding to the three detection methods, are shown in Fig. 2(a), for noise variance $\sigma_{\widetilde{n}}^{2}=1$. The optimal weights were found to be $\left(a_{0}, a_{1}, a_{2}, a_{3}\right)^{n}=(0.717,0.126,0.127,0.03)$. It is clearly shown that the proposed technique outperforms, by a significant margin, the conventional high-resolution and low-resolution watermark detection. It can be also seen that the experimentally obtained ROC curves remarkably coincide with the theoretical ones for all examined detection scenarios. The equal error rate (EER) points (the points on the ROC curve where the probability of false alarm equals the probability of false rejection), obtained from the corresponding ROC curves,

${ }^{2} H(\boldsymbol{z})$ denotes the $z$-transform of $h[\boldsymbol{m}]$.

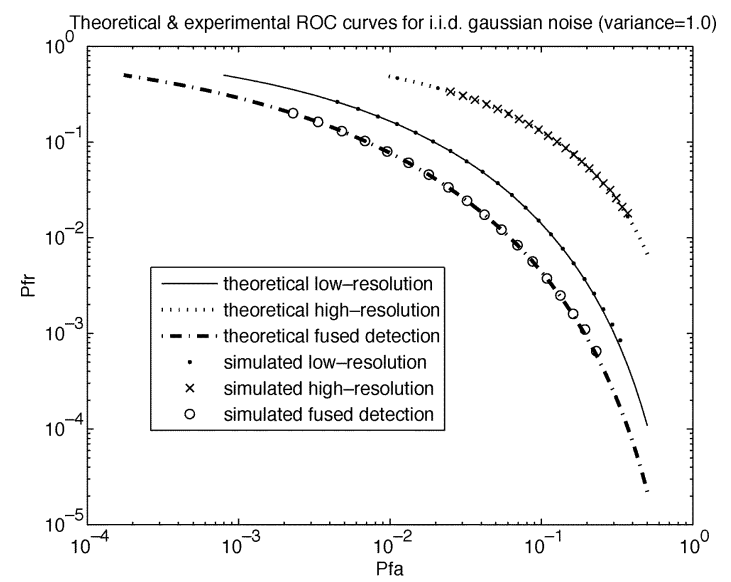

(a)

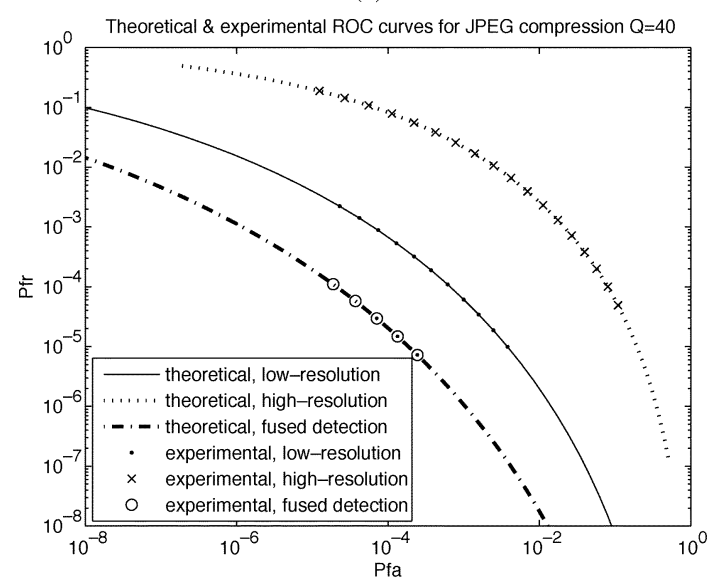

(b)

Fig. 2. Theoretical and experimental ROC curves for watermark detection in the low/high-resolution image and fused-correlation detection after: (a) additive i.i.d. gaussian noise of variance equal to 1 and (b) JPEG compression of quality $=40$.

are graphically presented in Fig. 3(a), for all three detection procedures and for various noise variances $\sigma_{\widetilde{n}}^{2}$. The proposed fused correlation-detection scheme is consistently superior to both alternative methodologies for all examined values of the noise variance. It should be noted that the EER improvement was found to be always greater than $15 \%$, compared to the low-resolution and greater than $36 \%$ compared to the high-resolution detection schemes.

In a typical application scenario, though, the interpolated image will go through one or multiple compression/decompression engines. Therefore, another set of experiments is presented, where the interpolated image was compressed using JPEG of various quality factors (larger quality factors correspond to better image quality). Both theoretical and experimental ROC curves, for the three methods under investigation, are presented in Fig. 2(b) for a JPEG quality factors equal to 40 (optimal fusion was employed: $\left.\left(a_{0}, a_{1}, a_{2}, a_{3}\right)=(0.4,0.273,0.289,0.038)\right)$. The experimentally derived ROC curves are again in remarkable agreement with the theoretical ones. The corresponding EER values for several JPEG qualities can be seen in Fig. 3(b). It should be noted that in the case of JPEG compression, the noise signal is not actually uncorrelated, resulting in suboptimal choice 


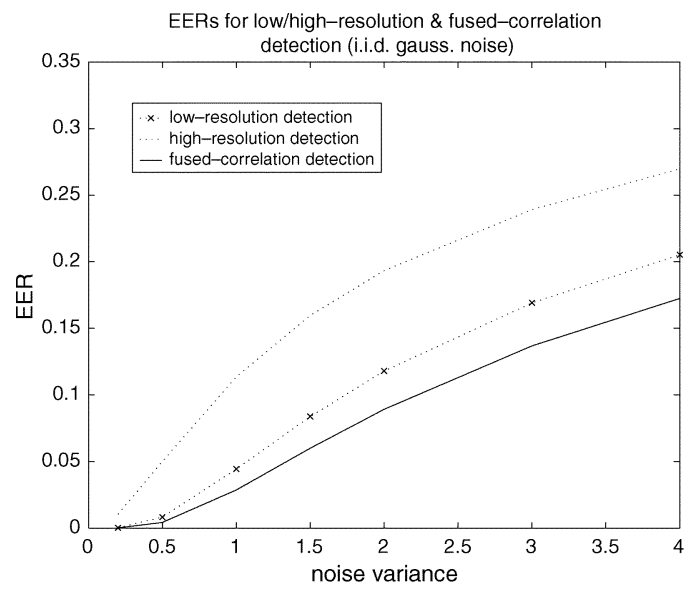

(a)

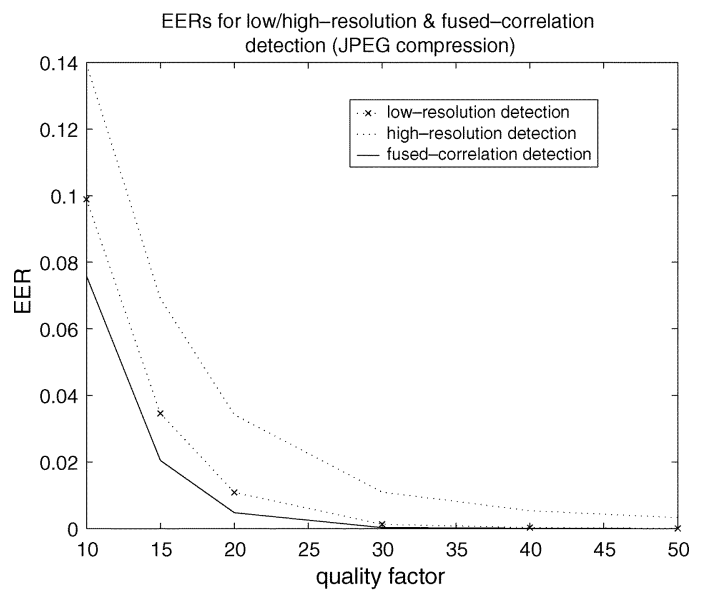

(b)

Fig. 3. EER values for: (a) additive i.i.d. Gaussian noise against various noise variances and (b) JPEG compression against various quality factors.

of the fusion weights $\left(a_{0}, a_{1}, \ldots, a_{M-1}\right)$. Even in this way, there is still considerable improvement, in comparison with the conventional watermark detection applied on the low-resolution image and even a much greater improvement, compared to the high-resolution detection scenario. In all cases, the improvement was measured to be greater than $22 \%$, compared to the low-resolution detection scheme and greater than $45 \%$ for the high-resolution case scenario, even if severe quality factors are imposed.

So far, it has been assumed that the scaling factor is an integer number. In the last set of experiments, noninteger scaling factors and alternative scaling transformations are examined. Specifically, using a commercial image processing software, the low-resolution watermarked image was scaled by a factor equal to 1.6 using bicubic interpolation and the resultant image was subjected to noise corruption (i.i.d. Gaussian noise of variance equal to 0.5 ), as described in the previous experiments. In order to apply our detection technique, the interpolated image was scaled again using bilinear interpolation, so that the final image reaches the closest (upward) integer factor, i.e., a factor equal to 2 in the particular examined case. By observing the corresponding ROC curves in Fig. 4, it is easily concluded that the

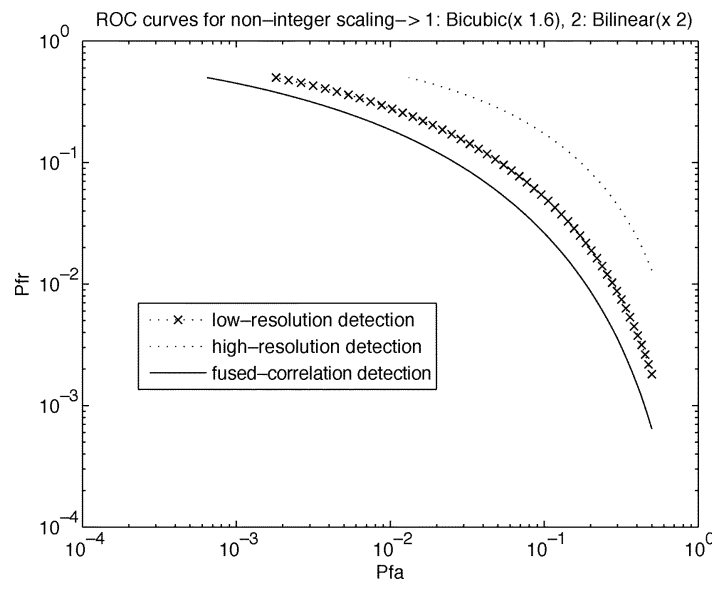

Fig. 4. ROC curves for bicubic noninteger initial scaling by 1.6 followed by bilinear rescaling to reach an integer factor equal to 2. Gaussian i.i.d. noise of variance 0.5 has been added.

proposed technique does not depend on any specific prior assumptions about the scaling factors and/or scaling methodologies and still outperforms the conventional detection techniques.

\section{CONCLUSION}

An efficient techniquewas used for improving the detection reliability of correlation-based image watermarking schemes in cases where noisy interpolated versions of the originally watermarked image are available for detection. The proposed fused correlation-detection scheme was theoretically shown and verified experimentally, to outperform traditional detection scenarios. Experimental results for both additive noise corruption and JPEG compression were provided.

\section{REFERENCES}

[1] S. Pereira and T. Pun, "Robust template matching for affine resistant image watermarks," IEEE Trans. Image Process., vol. 9, no. 6, pp. $1123-1129$, Jun. 2000

[2] M. Kutter, "Watermarking resisting to translation, rotation and scaling," in Proc. SPIE'98, vol. 2, Boston, MA, 1998, pp. 549-552.

[3] T. Kalker, J. P. Linnartz, and M. V. Dijk, "Watermark estimation through detector analysis," in Proc. ICIP'98, vol. 1, Chicago, IL, 1998, pp. 425-429.

[4] P. P. Vaidyanathan, "Multirate digital filters, filter banks, polyphase networks, and applications: a tutorial," Proc. IEEE, vol. 78, no. 1, pp. 56-93, Jan. 1990.

[5] J. P. Linnartz, T. Kalker, and G. Depovere, "Modeling the false alarm and missed detection rate for electronic watermarks," in Proc. 2nd Inf. Hiding Worksh., 1998, pp. 329-343.

[6] S. Tsekeridou, V. Solachidis, N. Nikolaidis, A. Nikolaidis, and I. Pitas, "Statistical analysis of a watermarking system based on bernoulli chaotic sequences," Signal Process., vol. 81, no. 6, pp. 1273-1293, 2001.

[7] T. Furon, B. Macq, N. Hurley, and G. Silvestre, "Janis: just another $n$-order side-informed watermarking scheme," in Proc. ICIP'02, Rochester, NY, 2002, pp. 153-156.

[8] D. Kundur and D. Hatzinakos, "Digital watermarking for telltale tamper proofing and authentication," Proc. IEEE, vol. 87, no. 7, pp. 1167-1180, Jul. 1999.

[9] P. Billingsley, Probability and Measure. New York: Wiley, 1995.

[10] D. P. Bertsekas, Constrained Optimization and Lagrange Multiplier Methods. New York: Academic, 1982. 